\title{
Prions of Yeast Are Genes Made of Protein: Amyloids and Enzymes
}

\author{
R.B. Wickner, H.K. Edskes, E.D. Ross, M.M. Pierce, F. ShewmakeR, \\ U. BAXA, AND A. BRACHMANN \\ Laboratory of Biochemistry and Genetics, National Institute of Diabetes, Digestive and Kidney Diseases, \\ National Institutes of Health, Bethesda, Maryland 20892-0830
}

In 1994 we described two infectious proteins (prions) of Saccharomyces cerevisiae, showing that the nonchromosomal genes [URE3] and [PSI] are inactive, self-propagating forms of Ure2p and Sup35p, respectively (Wickner 1994). Since then, [Het-s], a prion of Podospora anserina (Coustou et al. 1997), and [PIN+], another $S$. cerevisiae prion (Derkatch et al. 1997, 2001; Sondheimer and Lindquist 2000), have been found. All of the above prions are based on self-propagating amyloids. Recently, we described another prion, called $[\beta]$, which is based on the trans self-activation by the yeast vacuolar protease B (Roberts and Wickner 2003). Evidence has appeared suggesting that $\mathrm{C}$, the non-Mendelian gene of Podospora anserina determining Crippled Growth, is also a self-activating prion of a MAP kinase kinase kinase (Kicka and Silar 2004). Study of these yeast and fungal phenomena has established that proteins can be genes and infectious entities and has revealed many details of what makes a protein infectious.

\section{"EPIGENETICS" AND "PRIONS": WHAT DOES IT ALL MEAN?}

"Epigenetic" has been defined as ". . . heritable changes in gene function that cannot be explained by changes in DNA sequence" (Riggs and Porter 1997). The prions (infectious proteins) alter the expression of genes without changes in DNA sequence, but do so by altering the gene product, without necessarily altering the function of the gene itself. However, "epigenetic" is more broadly used to mean any heritable change not due to a change in nucleic acid sequence.

The notion of infectious proteins originated in attempts to explain the relative radiation resistance of the scrapie agent (Alper et al. 1967; Griffith 1967). The term "prion," which we now use to mean "infectious protein without a needed nucleic acid" (the protein-only model), was later coined as a synonym for the agent causing transmissible spongiform encephalopathies (TSEs), without requiring that this be its mechanism, but including this possibility (Prusiner 1982). In spite of two Nobel prizes, it seems that there is still doubt in some quarters about whether the TSEs are infectious proteins (Farquhar et al. 1998; Chesebro 2003; Priola et al. 2003). Our view is that there is considerable evidence pointing in this direction (see Wickner et al. 2004), but we remain agnostic about whether this is enough. Two potential "final proofs" have famously not yet worked: (a) amyloid formed in vitro from recombinant $\mathrm{PrP}$ is not infectious for normal mice, and (b) brain extracts of transgenic mice dying with a scrapie-like syndrome as a result of carrying PrP genes with mutations that cause inherited Creutzfeldt-Jakob disease (CJD) in humans are again not infectious for normal mice.

\section{FOUR TYPES OF PRION}

There are four known types of prion, depending on whether the altered form of the protein is an amyloid or not, and whether the prion is toxic to the individual, is simply inactive for its normal function, or positively helps the cell (Fig. 1). In the TSEs, assuming they are infectious proteins, the amyloid form of PrP is cytotoxic. The [URE3] and [PSI+] prions of yeast are simply inac-

\section{Four known prion types:}

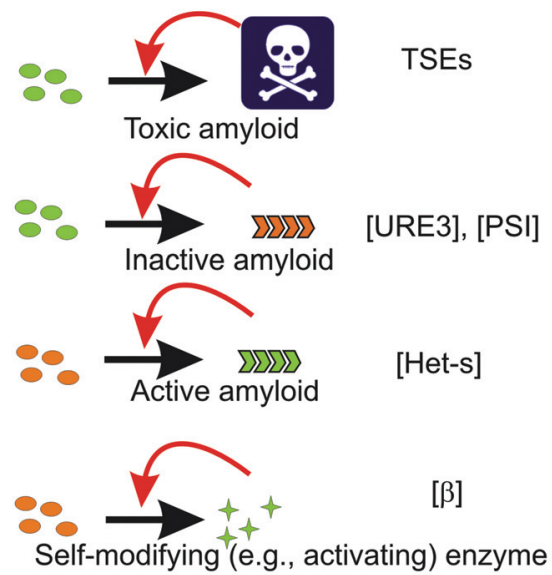

Figure 1. Four prion types. The known prions differ in whether they are based on amyloid or not, and what effect they have on the organism. The TSEs are lethal because of a novel harmful effect of the amyloid form of PrP. Cells with [URE3] and [PSI] partially lose the normal function of Ure $2 p$ and Sup35p, respectively. In [Het-s] strains, the HETs protein carries out a function for the cell only in the amyloid form (and is generally found in this form in wild isolates). The $[\beta]$ prion is simply the active form of the vacuolar protease $\mathrm{B}$, has no relation to amyloid, and functions for the cell in starvation and meiosis. 
tive (or partially inactive) amyloid forms of yeast Ure $2 p$ and Sup35p, respectively. [Het-s] of Podospora anserina is an amyloid-based prion that performs a function for the cell, helping in the heterokaryon incompatibility that this organism uses to avoid harmful infectious agents (such as viruses and mitochondrial senility factors) that spread by fusion of cellular processes (hyphal anastomosis). The $[\beta]$ prion of $S$. cerevisiae is not amyloid-based but is simply an enzyme whose active form is necessary in trans for activation of its own inactive precursor. [ $\beta$ ] functions for the cell in promoting survival under starvation conditons and allowing meiosis and spore formation.

\section{GENETIC CRITERIA FOR PRIONS}

The first yeast prions were discovered based on their paradoxical genetic properties, properties that are unknown (and unexpected) for nucleic acid replicons, but are expected for prions in which the phenotype produced is due to simple inactivation of the prion protein's normal function (Fig. 2) (Wickner 1994). These properties were emphatically not the observed properties of the TSEs, both because they were designed for a prion of a microorganism and because TSEs cannot be a consequence of simple inactivation of PrP, since deletion of the PRNP gene produces essentially no phenotype (Bueler et al. 1992).

\section{Reversible Curing}

If a prion can be cured, then it should be possible for it to arise again in the cured strain, because the protein with the potential to become the prion is still present. Reversible curing is to be distinguished from revertible mutation. Curing is the efficient elimination of a nonchromosomal gene by some treatment or condition that, for a nucleic acid replicon, would mean elimination of the replicon-generally an irreversible event. Mutation is generally not so efficient and can revert.

\section{Overproduction of the Protein Increases Frequency of Prion Generation}

More of the protein with the potential to become a prion should result in a higher frequency of de novo prion generation, regardless of the mechanism.

\section{Gene Required for Prion Propagation and Phenotype Relationship}

The same gene whose overexpression increases prion generation should be essential for prion propagation. Furthermore, for prions of the "inactive amyloid" type (see Fig. 1), the phenotype of the presence of the prion should resemble the phenotype of a recessive mutation in the gene, since in each case there is a deficiency of the normal form.

All three genetic criteria apply strictly to the prions of the inactive amyloid type, indeed to any prion whose phenotype is due to self-propagating inactivation of the nor-
Diagnostic Features of Prions:
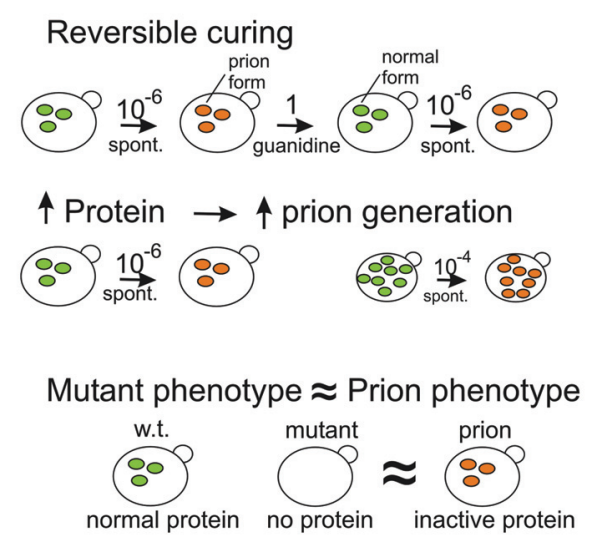

Figure 2. Genetic criteria for a prion. These three properties are expected of a nonchromosomal gene if it is a prion, but are not expected if it is a nucleic acid replicon (Wickner 1994).

mal form (whether by amyloid formation or not). The final genetic feature only half applies to other prions; the gene encoding the protein must be necessary for the propagation of the prion, but the phenotype relation will not be as described (Wickner 1994).

\section{[URE3] AND [PSI+] ARE PRIONS OF Ure2p AND Sup35p, RESPECTIVELY}

The non-Mendelian gene [URE3] and the chromosomal ure 2 mutant were discovered by Lacroute in the same genetic selection for strains that could take up ureidosuccinate to replace the absence of that intermediate in a $u$ ra 2 (aspartate transcarbamylase) mutant (USA+) (Lacroute 1971; Drillien and Lacroute 1972). The phenotypes of [URE3] and ure2 strains are thus similar, and, remarkably, ure2 mutants cannot propagate [URE3] (Drillien and Lacroute 1972; Aigle and Lacroute 1975; Wickner 1994). [URE3] can be cured by growth of cells in the presence of millimolar guanidine (Aigle and Lacroute 1975), but from the cured clones can again be isolated [URE3] subclones (Wickner 1994). Finally, overproduction of Ure2p (URE2 mRNA does not suffice) induces a 100-fold increase in the frequency with which [URE3] arises de novo (Wickner 1994). This showed that [URE3] is a prion of Ure $2 p$. When yeast is presented with a good nitrogen source, such as ammonia, glutamine, or glutamate, it represses the production of the enzymes and transporters needed to utilize poor nitrogen sources. Ure $2 p$ is a central player in this process, binding the Gln3p transcription factor and keeping it in the cytoplasm under repressing conditions (for review, see Cooper 2002).

$[\mathrm{PSI}+]$ is a nonchromosomal gene that increases the efficiency of weak non-sense suppressor tRNAs (Cox 1965; Cox et al. 1988). Results similar to those for [URE3] implied that [PSI+] is a prion of the Sup35 protein (Wickner 1994). [PSI+] is efficiently cured by growth in hyperosmotic media (Singh et al. 1979), but 
[PSI+] derivatives can again be isolated from cured cells (Lund and Cox 1981). Overproduction of Sup35p increases the frequency with which [PSI+] arises (Chernoff et al. 1993; Derkatch et al. 1996). Finally, the phenotype of the presence of [PSI] resembles that of recessive sup 35 mutants, and [PSI] propagation depends on the SUP35 gene (Doel et al. 1994; Ter-Avanesyan et al. 1994). Sup35p is a subunit of the translation termination factor (Frolova et al. 1994; Stansfield et al. 1995), explaining these effects on suppression of non-sense mutations.

These results showed that [URE3] and [PSI+] are prions of Ure2p and Sup35p (Wickner 1994), respectively; these conclusions have been amply confirmed by numerous later studies. For [URE3], it was shown that Ure2p becomes protease-resistant in [URE3] strains (Masison and Wickner 1995), and that this protease resistance was not a consequence of the altered nitrogen regulation (Masison et al. 1997). It is the Ure2 protein (not the mRNA or DNA gene) whose overproduction induces the de novo appearance of [URE3] (Masison and Wickner 1995; Masison et al. 1997), a result that may well be sufficient to prove a prion. Sup35p is aggregated in $[\mathrm{PSI}+]$ cells (Patino et al. 1996; Paushkin et al. 1996) and it is the protein, not the RNA or gene, that induces [PSI+] to arise de novo (Derkatch et al. 1996).

\section{THE AMINO TERMINUS OF URe2p CONSTITUTES A PRION DOMAIN}

Deletion analysis of Ure2p showed that the amino-terminal 65 residues were sufficient to induce the appearance of [URE3] (Masison and Wickner 1995). This region was also sufficient to propagate [URE3] in the complete absence of the carboxy-terminal part of the molecule (Masison et al. 1997). In contrast, the carboxy-terminal residues 66-354 (Coschigano and Magasanik 1991; Masison and Wickner 1995) or even residues 90-354 (Maddelein and Wickner 1999) are sufficient to carry out the nitrogen regulation function of Ure2p (Fig. 3). These results indicated that the interaction between Ure2p molecules that propagated the [URE3] prion were between one amino-terminal prion domain and another, and that the carboxy-terminal part of the molecule was only the indicator of the prion change, but not its mediator.

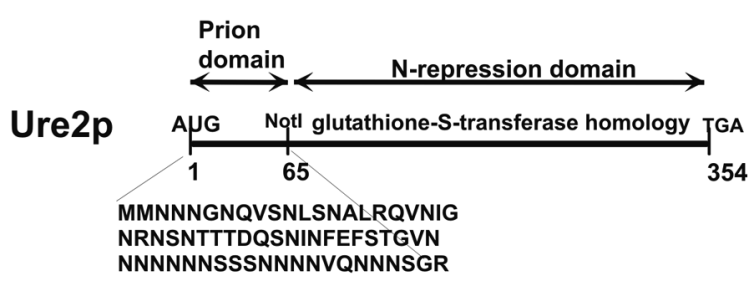

Figure 3. Prion domain of Ure2p. Deletion analysis showed that residues $1-65$ are sufficient to induce [URE3] prion formation and necessary for Ure $2 p$ to participate in the prion change (Masison and Wickner 1995; Masison et al. 1997). This region can also propagate [URE3] in the absence of the carboxy-terminal domain. Residues 66-354 can carry out the nitrogen regulation function of Ure2p (Masison and Wickner 1995).

\section{THE [URE3] PRION IS A SELF-PROPAGATING AMYLOID FORM OF Ure2p}

The protease-resistance of Ure2p in [URE3] strains (Masison and Wickner 1995), and the appearance of Ure2GFP fusion protein in [URE3] cells (Edskes et al. 1999a) suggested that the [URE3] change of Ure2p involved aggregation. Chemically synthesized Ure $2 \mathrm{p}^{1-65}$, the prion domain peptide, spontaneously forms amyloid in vitro, suggesting a role for this abnormal protein form in the [URE3] prion (Taylor et al. 1999). Just as overproduced Ure $2 \mathrm{p}^{1-65}$ induces the full-length protein to undergo the prion change in vivo, the same prion domain peptide induces amyloid formation by the otherwise stably soluble full length Ure2p in vitro (Taylor et al. 1999). This amyloid formation could be further propagated by addition of more native Ure $2 p$. The pattern of protease-resistant bands of Ure2p seen in extracts of [URE3] cells (Masison and Wickner 1995) is identical to that seen for amyloid of Ure2p formed in vitro (Taylor et al. 1999). In each case, the most protease-resistant part of the molecule is the prion domain itself(Masison and Wickner 1995; Taylor et al. 1999; Baxa et al. 2003). It is possible to detect networks of filaments specifically in [URE3]-carrying cells (Speransky et al. 2001). Using antibody to Ure2p linked to gold particles, it was shown that these filaments contained Ure2p.

\section{ARCHITECTURE OF AMYLOID FILAMENT FORMED FROM Ure2p}

The fact that the prion domain could propagate the [URE3] prion in the complete absence of the carboxy-terminal domain (Masison et al. 1997) implies that the primary interaction between molecules in the prion (amyloid) form of Ure $2 p$ is between one amino-terminal prion domain and another. This conclusion was confirmed by showing that protease digestion of filaments formed in vitro or Ure2p in extracts of [URE3] cells leaves a protease-resistant core consisting of the prion domain. The filaments become more narrow on digestion and are similar to those formed from the synthetic prion domain peptide (Taylor et al. 1999; Baxa et al. 2003).

The carboxy-terminal functional domain is only inactivated by prion formation if it is covalently attached to the prion domain (Masison et al. 1997). This could mean that the carboxy-terminal domain is conformationally changed by association with the amyloid-forming prion domain, or that amyloid formation makes the carboxyterminal domain sterically unable to interact with $G \ln 3 p$, its normal function. A series of fusion proteins were prepared consisting of the amino-terminal prion domain linked to enzymes whose structures are known and whose substrates are too small to be subject to steric hindrance by amyloid formation. Each of these fusion proteins readily formed amyloid filaments and in each case the enzyme was fully active in the amyloid filaments. This surprising result is particularly significant in the case of the Ure $2 p^{1-65}$-glutathione-S-transferase fusion protein because the carboxy-terminal part of Ure $2 p$ is homologous to GSTs in both sequence and structure (Coschigano and Magasanik 1991; Bousset et al. 2001; Umland et al. 


\section{Ure2p Amyloid Architecture:}

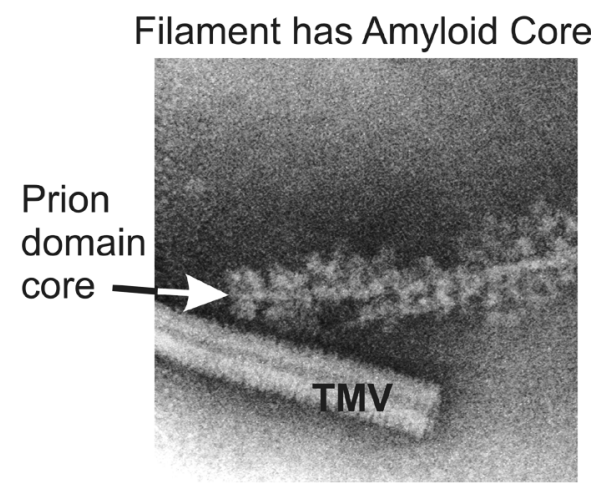

Figure 4. Architecture of amyloid filaments of Ure2p. Scanning transmission electron micrograph of a Ure $2 p$ amyloid filament showing the central core of amyloid, composed of the prion domain, and the appended carboxy-terminal domain (Baxa et al. 2003).

2001). This indicates that the carboxy-terminal domain of Ure $2 p$ is not conformationally changed in the prion (amyloid) form, but is unable to interact with Gln3p nonetheless. One intriguing possible explanation (Baxa et al. 2002 ) is that Gln $3 p$ association with Ure $2 p$ is a diffusionlimited reaction that is inefficient when Ure $2 p$ is restricted to a small part of the cytoplasm in the network of filaments (Speransky et al. 2001).

Scanning transmission electron microscopic images of Ure $2 p$ amyloid filaments show that each has a central core around which is arrayed larger masses attached rather loosely to the central core (Fig. 4) (Baxa et al. 2003). The central core of these filaments was shown by Western blot and mass spectrometry to be comprised of Ure $2 p$ residues 1-65 (with some fraying in some molecules) (Baxa et al. 2003). The masses arrayed around the core are the GSTlike carboxy-terminal domains, consisting of residues

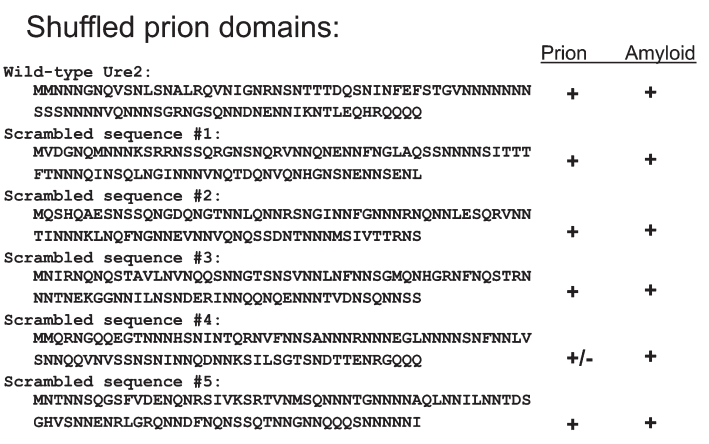

Conclusion: For the Ure2 prion domain, prion-forming ability is determined by amino acid content, not by amino acid sequence.

Figure 5. Shuffled prion domains can be prions and form amyloid. Residues 1-89 of Ure $2 p$ were randomly ordered, and replaced the normal sequence at the normal URE2 chromosomal locus. The amino acid content was unchanged, but the sequence was completely random. Ability of each of these five randomized proteins to form prions in vivo and amyloid in vitro was tested and each was capable of both (Ross et al. 2004).
95-354. The carboxy-terminal domains are connected to the amyloid cores by a tether comprising roughly residues 70-95. This region remains highly protease-sensitive in the amyloid form, when both the prion domain and carboxy-terminal domain are structured (Baxa et al. 2003).

The structure of the amyloid core remains to be determined. The recent demonstration by Tycko and coworkers of a parallel $\beta$-sheet structure of the $A \beta$ peptide (Petkova et al. 2002; Tycko 2003) suggests that a similar structure may be found for the Ure2p prion domain amyloid. All Ure2p amyloid filaments have one monomer per $0.45 \mathrm{~nm}$, consistent with this type of structure (Baxa et al. 2003).

\section{AMINO ACID COMPOSITION IS THE MAIN DETERMINANT OF Ure2p PRION FORMATION}

The N/Q-rich nature of the Ure $2 p$ and Sup35p prion domains (Ter-Avanesyan et al. 1994; Masison and Wickner 1995) and the demonstrated importance of Q residues in Sup35p (DePace et al. 1998) and runs of $N$ in Ure $2 p$ (Maddelein and Wickner 1999) implicate at least this feature as important in prion formation. However, there are over 100 yeast proteins with N/Q-rich regions, and, aside from Ure2p and Sup35p, only Rnq1p has been shown to be a prion (Derkatch et al. 1997, 2001; Sondheimer and Lindquist 2000). To determine whether there were sequence requirements for prion (or amyloid) formation by Ure $2 p$, we constructed a series of derivatives in which the amino acids of the prion domain of Ure $2 p$ had been randomly shuffled without changing the overall composition or codon usage (Fig. 5). We found that all five such random sequences were capable of becoming prions in vivo and the purified proteins were able to form amyloid in vitro (Ross et al. 2004). This shows that, in the case of Ure $2 p$, the major factor determining prion (and amyloid) formation is amino acid composition. There were differences in how robust were the prions formed by the various shuffled derivatives, showing that sequence is indeed a lesser factor but a factor nonetheless (Ross et al. 2004). Future studies will determine whether other prion domains can be shuffled without loss of activity, what aspects of composition (aside from N/Q richness) are important, and what are the sequence determinants of prion robustness.

\section{CHAPERONES INVOLVED IN [URE3] PROPAGATION}

That $[\mathrm{PSI}+]$ requires the disaggregating chaperone Hsp104 for its propagation and the curing of [PSI+] by overproduction of Hsp104 were crucial findings, opening up a vital new area in prion (and amyloid) studies (Chernoff et al. 1995). In addition, guanidine curing of yeast prions has been shown to occur by inhibition of Hsp104 (Ferreira et al. 2001; Jung and Masison 2001; Jung et al. 2002). Guanidine treatment was used as a tool to study the mechanism of Hsp104's contribution to [PSI+] propagation. It was found that guanidine treatment had no immediate effect on the incorporation of newly synthesized Sup35p into aggregates, but instead blocked the forma- 
tion of new prion seeds (Ness et al. 2002; Tuite and Cox 2003). Hsp70s have also been implicated in prion propagation (Chernoff et al. 1999; Newnam et al. 1999; Jung et al. 2000; Jones and Masison 2003). Likewise for the Hsp40 family and various cochaperones (Kushnirov et al. 2000; Jones et al. 2004).

We find that propagation of [URE3] requires Hsp104, but that, unlike [PSI+], overproduction of Hsp104 does not cure [URE3] (Moriyama et al. 2000). However, overproduction of Ydj1p, an Hsp40 family member, does cure [URE3] (Moriyama et al. 2000). Mutation of SSA2, encoding the most abundant of the cytoplasmic Hsp70s, also results in loss of [URE3] (Roberts et al. 2004). Interestingly, overexpression of Ssa1p eliminates [URE3], although identical overexpression of Ssa2p does not (Schwimmer and Masison 2002).

\section{GENETIC CONTROL OF [URE3] GENERATION: Mks1p AND [PIN+]}

Our finding that overproduction of Mks1p negatively affects the ability of Ure $2 p$ to regulate nitrogen catabolism (Edskes et al. 1999b) suggested the possibility that Mks1p might likewise affect prion generation or propagation. We found that $m k s 1 \Delta$ strains were nearly unable to generate [URE3] derivatives, even when Ure2p or one of its highly prion-inducing derivatives was overexpressed (Edskes and Wickner 2000). Modest overproduction of Mks1p resulted in a substantial increase in [URE3] prion generation. The same $m k s 1 \Delta$ strains were fully competent to propagate [URE3] and to show the USA+ phenotype typical of [URE3] strains (Edskes and Wickner 2000). This shows that the effect of $m k s 1 \Delta$ on prion formation was an effect on prion generation, not an effect on propagation or detection of the [URE3] prion.

$M K S 1$ was first defined as a gene whose overexpression slows growth, an effect antagonized by the $R A S$ cAMP pathway (Matsuura and Anraku 1993). We found that expression of the constitutive ("oncogenic") Ras ${ }^{\text {val19 }}$ mutant dramatically inhibited [URE3] generation, as one would expect if it inhibited Mks1p action (Edskes and Wickner 2000).

The $[\mathrm{PIN}+]$ nonchromosomal genetic element was discovered as a factor necessary for [PSI + ] inducibility on overproduction of Sup35p (Derkatch et al. 1997). Subsequent genetic analysis showed that $[\mathrm{PIN}+]$ is a prion of the Rnq1 protein, a nonessential protein rich in asparagine and glutamine residues throughout most of its length (Derkatch et al. 2000, 2001; Sondheimer and Lindquist 2000). The [PIN+] effect may be a result of cross-seeding between $\mathrm{Q} / \mathrm{N}$-rich amyloid formed by Rnq1 and the prion domain of Sup35p. A similar, but less dramatic, effect is seen with [URE3]. [PIN+] strains are 10- to 100-fold more frequently induced to form [URE3] than are isogenic [pin-] strains (Bradley et al. 2002).

\section{Ure2 HOMOLOGS IN PATHOGENIC YEASTS}

Although Ure2p is weakly homologous to many GSTs, and it has not been found to have GST activity in vitro, it is reported to confer a low level of metal resistance (Rai et al. 2003). However, close relatives of Ure $2 p$ are found in a series of yeasts, including other species of Saccharomyces, Schizosaccharomyces pombe, Candida albicans, Candida lypolytica, Candida maltosa, Ashbya gossypii, and others (Edskes and Wickner 2002). Most of these foreign URE2 genes were able to complement an $S$. cerevisiae ure $2 \Delta$ mutant, reflecting the $80-100 \%$ identity in their carboxy-terminal domains (Edskes and Wickner 2002). Their amino-terminal domains show far lower similarity, and, in the case of Saccharomyces pombe, no amino-terminal domain at all. Those amino-terminal extensions are all $\mathrm{Q} / \mathrm{N}$-rich, suggesting some utility for the prion domain. Indeed, expression of only the carboxy-terminal domain gives a leaky phenotype, with some expression of normally nitrogen-repressed genes (Masison and Wickner 1995). Since [URE3] and ure 2 mutant cells grow slowly on most media, it is likely that the evolutionarily selected function of the Ure $2 p$ prion domain is not for the rare prion formation but rather for the promotion of the normal function of Ure $2 p$. However, there is at least one case of a prion that is functional for its host, the [Het-s] prion of Podospora.

\section{[Het-s], AN ADAPTIVE AMYLOID PRION OF PODOSPORA ANSERINA}

When two colonies of a filamentous fungus meet, they fuse their cellular processes (hyphae) perhaps to share nutrients, but only if they are genetically identical. Genetic identity is tested by checking identity at around a dozen polymorphic loci scattered around the genome. These are call het loci in Podospora and include het-s, whose alleles are het-s and het-S. When colonies meet, a few hyphae fuse in a sort of trial. If they differ in alleles at even a single het locus, these fused hyphae degenerate and die and set up a barrier to further fusions. This reaction is called "heterokaryon incompatibility." The het-s locus encodes a 289-amino-acid protein differing at 13 residues between the het-s and het-S alleles.

Studies by Saupe and colleagues at Bordeaux have shown that only if the het-s protein is in a prion form is the proper incompatibility reaction observed (Coustou et al. 1997; Saupe et al. 2000; Maddelein et al. 2002). This prion is called [Het-s] and is based on a self-propagating amyloid form of the protein encoded by the het- $s$ allele. Since heterokaryon incompatibility is a normal fungal function, possibly designed to prevent the spread of debilitating fungal viruses and plasmids between unrelated strains, it is reasonable to assume that this prion is carrying out a function for the cell. This hypothesis is supported by the fact that essentially all isolates of $\mathrm{Po}$ dospora with the het-s allele carry the prion.

All yeast and fungal prions are nonchromosomal genes that spread via cell-cell fusion such as occurs in sexual matings or in nonsexual matings such as the hyphal anastomosis discussed above for Podospora. This infectious mode of spread means that these elements should gradually invade the population unless they are a disadvantage to their hosts. Thus, finding a prion in the wild does not imply it is an advantage, but failure to find it in the wild is a strong indication that the prion is a disadvantage to 


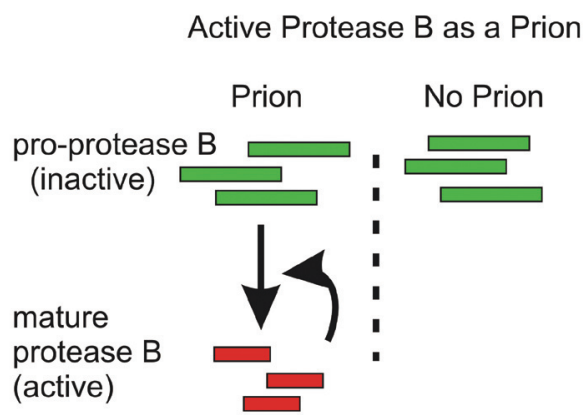

MAP kinase kinase kinase as a Prion

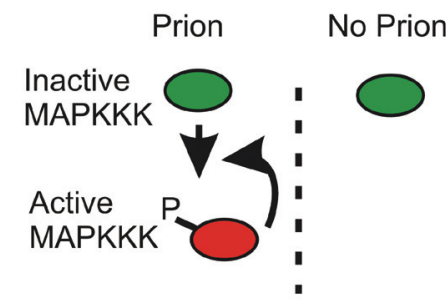

Figure 6. A new type of prion: an enzyme needed for its own activation. Vacuolar protease B of $S$. cerevisiae can be a prion in the absence of protease A, which normally catalyzes its activation (Roberts and Wickner 2003). The properties of the "C" nonchromosomal gene of the filamentous fungus Podospora anserina suggest that it too may be a prion of this type, based on self-activation of a MAP kinase kinase kinase (Kicka and Silar 2004). It is possible that the MAP KKK activation is done by one of the kinases further down in the cascade, MAP KK or MAP K.

the host. For example, two efforts to find the [PSI+] prion in wild strains have been unsuccessful (Chernoff et al. 2000; Resende et al. 2002), indicating that this prion is, on the net, a disadvantage to the host.

\section{[ß], A DIFFERENT KIND OF PRION}

The word prion means "infectious protein," and, as first discussed by Griffith (Griffith 1967), need not involve amyloid. If the active (or, more generally, modified) form of an enzyme is necessary for its own activation (or modification), then a cell that starts out without the active (modified) form will never develop it unless it comes from outside the cell. A cell with the active (modified) form will continue to activate (modify) newly synthesized protein molecules and should pass the trait "has active enzyme" on to its offspring. Transmission of the active protein to a cell lacking it should likewise transmit the self-propagating trait as well (Roberts and Wickner 2003).

Based on extensive work by Beth Jones (Carnegie Mellon University; Zubenko and Jones 1982) and Deiter Wolf (University of Stuttgart; Teichert et al. 1989), we have identified such a prion-namely, the active form of the vacuolar protease B of $S$. cerevisiae encoded by the PRB1 gene (Fig. 6) (Roberts and Wickner 2003). Protease $\mathrm{B}$, encoded by $P R B 1$, is made as an inactive precursor, whose activation by deletion of amino-terminal and carboxy-terminal extensions is carried out in normal cells by combined action of itself and protease A (for re- view, see Jones 1991). However, in mutants deleted for

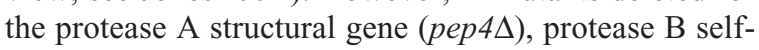
activation can continue indefinitely as cells grow (Roberts and Wickner 2003). The active state can be transmitted by cytoplasmic mixing from a cell with active protease B to one lacking it, showing that this trait is a nonchromosomal gene. The state in which cells have protease $B$ activity is called $[\beta]$ and its absence $[\beta-o]$.

$[\beta]$ has the characteristics expected of a prion for which the prion form is the active form of the protein. Cells that start out with protease B activity give rise to offspring, nearly all of which have active enzyme. Such a strain is efficiently cured of protease B activity by growth on rich dextrose media, a condition that partially represses $P R B 1$ transcription, leading to the self-activation cycle rapidly dying out. But of these cured cells, about 1 in $10^{5}$ descendants has spontaneously developed protease B activity (reversible curing). Overexpression of $P R B 1$ increases this frequency to several percent (genetic criterium 2 for a prion) (Roberts and Wickner 2003). [ $\beta$ ] depends on PRB1 for its propagation, but, of course, the phenotype of $[\beta]$ is not the same as a prbl mutant because the prion form is the active form of the protein.

$[\beta]$ is advantageous to the cell, since $[\beta]$ cells can undergo meiosis, while $[\beta-o]$ cells cannot, and cells with $[\beta]$ survive starvation better than do those without (Roberts and Wickner 2003).

The importance of the $[\beta]$ prion lies not in its biochemical mechanism, which has been recognized for decades, but in the facts that (1) a protease can be a gene and (2) there are many potentially self-modifying enzymes (protein kinases, protein acetylases, protein methylases, etc.) that, under some circumstances, might become prions. Indeed, recent work on the Crippled Growth phenomenon of Podospora anserina appears to be just such a new prion (Kicka and Silar 2004).

\section{CRIPPLED GROWTH AS A NEW PRION OF PODOSPORA ANSERINA}

Crippled growth (CG) is a condition that can develop in Podospora which is characterized by increased pigmentation and slowed growth (Silar et al. 1999). CG is the result of carrying the nonchromosomal gene "C." If a piece of a colony with $\mathrm{CG}$ is placed on a non-CG colony, heterokaryon formation leads to cytoplasmic mixing and the non-CG colony acquires the $\mathrm{CG}$ trait and the $\mathrm{C}$ genetic element.

Recent work has shown that the propagation of $\mathrm{C}$ (not just the phenotype, but the cytoplasmic gene) requires a MAP kinase kinase kinase similar to that encoded by $B C K 1$ of $S$. cerevisiae, and called PaASK1 because of its homology to human ASK1 (Kicka and Silar 2004). Overexpression of PaASK1 results in generation of the $\mathrm{C}$ element under conditions where its formation would be rare. $\mathrm{C}$ can be cured, but will arise anew in the cured strains. The phenotype of $\mathrm{C}$ is different from that of mutants in PaASK1, indicating that it is not inactivity of PaASK1 that produces C (Kicka and Silar 2004). While evidence of altered activity of the kinase cascade in C-carrying strains will be needed, it seems likely that $\mathrm{C}$ is a new case of a prion of the same type as $[\beta]$ (Fig. 6). As previously sug- 
gested by Silar (Silar and Daboussi 1999), filamentous fungi may prove to be a rich source of prions. Their syncytial structure allows newly arisen prions to spread throughout the colony, and heterokaryon formation provides a rapid route for infection of neighbors.

\section{CONCLUSIONS}

The discovery of yeast prions has ended the debate about whether there can be such a thing as a prion, although it has not ended the debate about whether TSEs are such entities. The ease of genetic manipulation of yeast has led to identification of a number of cellular components affecting the generation and propagation of prions, and produced a great deal of insight into prion phenomena, long pioneered in the more difficult animal systems. The new class of nonamyloid prions (now that there are apparently two of them, we are justified in calling it a "class") expands the possibilities for "infectious proteins" to a host of new mechanisms. It is expected that yeast and other fungi will continue to be useful in revealing the range of prion phenomena and their mechanisms.

\section{REFERENCES}

Aigle M. and Lacroute F. 1975. Genetical aspects of [URE3], a non-Mendelian, cytoplasmically inherited mutation in yeast. Mol. Gen. Genet. 136: 327.

Alper T., Cramp W.A., Haig D.A., and Clarke M.C. 1967. Does the agent of scrapie replicate without nucleic acid? Nature 214: 764.

Baxa U., Speransky V., Steven A.C., and Wickner R.B. 2002. Mechanism of inactivation on prion conversion of the Saccharomyces cerevisiae Ure2 protein. Proc. Natl. Acad. Sci. 99: 5253.

Baxa U., Taylor K.L., Wall J.S., Simon M.N., Cheng N., Wickner R.B., and Steven A. 2003. Architecture of Ure2p prion filaments: The N-terminal domain forms a central core fiber. $J$. Biol. Chem. 278: 43717.

Bousset L., Beirhali H., Janin J., Melki R., and Morera S. 2001. Structure of the globular region of the prion protein Ure2 from the yeast Saccharomyces cerevisiae. Structure 9: 39.

Bradley M.E., Edskes H.K., Hong J.Y., Wickner R.B., and Liebman S.W. 2002. Interactions among prions and prion "strains" in yeast. Proc. Natl. Acad. Sci. (suppl. 4) 99: 16392.

Bueler H., Fischer M., Lang Y., Bluethmann H., Lipp H.P., DeArmond S.J., Prusiner S.B., Aguet M., and Weissmann C. 1992. Normal development and behavior of mice lacking the neuronal cell-surface PrP protein. Nature 356: 577.

Chernoff Y.O., Derkach I.L., and Inge-Vechtomov S.G. 1993. Multicopy SUP35 gene induces de-novo appearance of psilike factors in the yeast Saccharomyces cerevisiae. Curr. Genet. 24: 268.

Chernoff Y.O., Lindquist S.L., Ono B.-I., Inge-Vechtomov S.G., and Liebman S.W. 1995. Role of the chaperone protein Hsp104 in propagation of the yeast prion-like factor [psi $\left.{ }^{+}\right]$. Science 268: 880 .

Chernoff Y.O., Newnam G.P., Kumar J., Allen K., and Zink A.D. 1999. Evidence for a protein mutator in yeast: Role of the Hsp70-related chaperone Ssb in formation, stability and toxicity of the [PSI+] prion. Mol. Cell. Biol. 19: 8103 .

Chernoff Y.O., Galkin A.P., Lewitin E., Chernova T.A., Newnam G.P., and Belenkly S.M. 2000. Evolutionary conservation of prion-forming abilities of the yeast Sup35 protein. Mol. Microbiol. 35: 865.

Chesebro B. 2003. Introduction to the transmissible spongiform encephalopathies or prion diseases. Br. Med. Bull. 66: 1 .

Cooper T.G. 2002. Transmitting the signal of excess nitrogen in Saccharomyces cerevisiae from the Tor proteins to the GATA factors: Connecting the dots. FEMS Microbiol. Rev. 26: 223.
Coschigano P.W. and Magasanik B. 1991. The URE2 gene product of Saccharomyces cerevisiae plays an important role in the cellular response to the nitrogen source and has homology to glutathione S-transferases. Mol. Cell. Biol. 11: 822 .

Coustou V., Deleu C., Saupe S., and Begueret J. 1997. The protein product of the het-s heterokaryon incompatibility gene of the fungus Podospora anserina behaves as a prion analog. Proc. Natl. Acad. Sci. 94: 9773.

Cox B.S. 1965. PSI, a cytoplasmic suppressor of super-suppressor in yeast. Heredity 20: 505.

Cox B.S., Tuite M.F., and McLaughlin C.S. 1988. The Psi factor of yeast: A problem in inheritance. Yeast 4: 159.

DePace A.H., Santoso A., Hillner P., and Weissman J.S. 1998. A critical role for amino-terminal glutamine/asparagine repeats in the formation and propagation of a yeast prion. Cell 93: 1241 .

Derkatch I.L., Bradley M.E., Hong J.Y., and Liebman S.W. 2001. Prions affect the appearance of other prions: The story of [PIN]. Cell 106: 171.

Derkatch I.L., Bradley M.E., Zhou P., Chernoff Y.O., and Liebman S.W. 1997. Genetic and environmental factors affecting the de novo appearance of the $[\mathrm{PSI}+]$ prion in Saccharomyces cerevisiae. Genetics 147: 507.

Derkatch I.L., Chernoff Y.O., Kushnirov V.V., Inge-Vechtomov S.G., and Liebman S.W. 1996. Genesis and variability of $[P S I]$ prion factors in Saccharomyces cerevisiae. Genetics 144: 1375 .

Derkatch I.L., Bradley M.E., Masse S.V., Zadorsky S.P., Polozkov G.V., Inge-Vechtomov S.G., and Liebman S.W. 2000. Dependence and independence of $\left[P S I^{+}\right]$and $\left[P I N^{+}\right]: A$ two-prion system in yeast? EMBO J. 19: 1942.

Doel S.M., McCready S.J., Nierras C.R., and Cox B.S. 1994. The dominant PNM2- mutation which eliminates the [PSI] factor of Saccharomyces cerevisiae is the result of a missense mutation in the SUP35 gene. Genetics 137: 659.

Drillien R. and Lacroute F. 1972. Ureidosuccinic acid uptake in yeast and some aspects of its regulation. J. Bacteriol. 109: 203.

Edskes H.K. and Wickner R.B. 2000. A protein required for prion generation: [URE3] induction requires the Ras-regulated Mks1 protein. Proc. Natl. Acad. Sci. 97: 6625.

2002. Conservation of a portion of the $S$. cerevisiae Ure2p prion domain that interacts with the full-length protein. Proc. Natl. Acad. Sci. (suppl. 4) 99: 16384.

Edskes H.K., Gray V.T., and Wickner R.B. 1999a. The [URE3] prion is an aggregated form of Ure $2 p$ that can be cured by overexpression of Ure2p fragments. Proc. Natl. Acad. Sci. 96: 1498.

Edskes H.K., Hanover J.A., and Wickner R.B. 1999b. Mks1p is a regulator of nitrogen catabolism upstream of Ure2p in Saccharomyces cerevisiae. Genetics 153: 585.

Farquhar C.F., Somerville R.A., and Bruce M.E. 1998. Straining the prion hypothesis. Nature 391: 345.

Ferreira P.C., Ness F., Edwards S.R., Cox B.S., and Tuite M.F. 2001. The elimination of the yeast [PSI+] prion by guanidine hydrochloride is the result of Hsp104 inactivation. Mol. Microbiol. 40: 1357.

Frolova L., LeGoff X., Rasmussen H.H., Cheperegin S., Drugeon G., Kress M., Arman I., Haenni A.-L., Celis J.E., Philippe M., Justesen J., and Kisselev L. 1994. A highly conserved eukaryotic protein family possessing properties of polypeptide chain release factor. Nature 372: 701.

Griffith J.S. 1967. Self-replication and scrapie. Nature 215: 1043.

Jones E.W. 1991. Three proteolytic systems in the yeast Saccharomyces cerevisiae. J. Biol. Chem. 266: 7963.

Jones G.W. and Masison D.C. 2003. Saccharomyces cerevisiae Hsp70 mutations affect $[\mathrm{PSI}(+)]$ prion propagation and cell growth differently and implicate Hsp40 and tetratricopeptide repeat cochaperones in impairment of $[\mathrm{PSI}(+)]$. Genetics 163: 495.

Jones G., Song Y., Chung S., and Masison D.C. 2004. Propagation of yeast $[\mathrm{PSI}+]$ prion impaired by factors that regulate Hsp70 substrate binding. Mol. Cell. Biol. 24: 3928. 
Jung G. and Masison D.C. 2001. Guanidine hydrochloride inhibits Hsp104 activity in vivo: A possible explanation for its effect in curing yeast prions. Curr. Microbiol. 43: 7.

Jung G., Jones G., and Masison D.C. 2002. Amino acid residue 184 of yeast Hsp104 chaperone is critical for prion-curing by guanidine, prion propagation, and thermotolerance. Proc. Natl. Acad. Sci. 99: 9936.

Jung G., Jones G., Wegrzyn R.D., and Masison D.C. 2000. A role for cytosolic $\mathrm{Hsp} 70$ in yeast [PSI+] prion propagation and [PSI+] as a cellular stress. Genetics 156: 559.

Kicka S. and Silar P. 2004. PaASK1, a mitogen-activated protein kinase kinase kinase that controls cell degeneration and cell differentiation in Podospora anserina. Genetics 166: 1241.

Kushnirov V.V., Kryndushkin D.S., Boguta M., Smirnov V.N., and Ter-Avanesyan M.D. 2000. Chaperones that cure yeast artificial $[\mathrm{PSI}+]$ and their prion-specific effects. Curr. Biol. 10: 1443 .

Lacroute F. 1971. Non-Mendelian mutation allowing ureidosuccinic acid uptake in yeast. J. Bacteriol. 106: 519.

Lund P.M. and Cox B.S. 1981. Reversion analysis of [psi-] mutations in Saccharomyces cerevisiae. Genet. Res. 37: 173.

Maddelein M.-L. and Wickner R.B. 1999. Two prion-inducing regions of Ure2p are non-overlapping. Mol. Cell. Biol. 19: 4516.

Maddelein M.-L., Dos Reis S., Duvezin-Caubet S., CoularySalin B., and Saupe S.J. 2002. Amyloid aggregates of the HET-s prion protein are infectious. Proc. Natl. Acad. Sci. 99: 7402.

Masison D.C. and Wickner R.B. 1995. Prion-inducing domain of yeast Ure $2 p$ and protease resistance of Ure $2 p$ in prion-containing cells. Science 270: 93.

Masison D.C., Maddelein M.-L., and Wickner R.B. 1997. The prion model for [URE3] of yeast: Spontaneous generation and requirements for propagation. Proc. Natl. Acad. Sci. 94: 12503.

Matsuura A. and Anraku Y. 1993. Characterization of the MKS1 gene, a new negative regulator of the ras-cyclic AMP pathway in Saccharomyces cerevisiae. Mol. Gen. Genet. 238: 6.

Moriyama H., Edskes H.K., and Wickner R.B. 2000. [URE3] prion propagation in Saccharomyces cerevisiae: Requirement for chaperone Hsp104 and curing by overexpressed chaperone Ydj1p. Mol. Cell. Biol. 20: 8916.

Ness F., Ferreira P., Cox B.S., and Tuite M.F. 2002. Guanidine hydrochloride inhibits the generation of prion "seeds" but not prion protein aggregation in yeast. Mol. Cell. Biol. 22: 5593 .

Newnam G.P., Wegrzyn R.D., Lindquist S.L., and Chernoff Y.O. 1999. Antagonistic interactions between yeast chaperones Hsp104 and Hsp70 in prion curing. Mol. Cell. Biol. 19: 1325.

Patino M.M., Liu J.-J., Glover J.R., and Lindquist S. 1996. Support for the prion hypothesis for inheritance of a phenotypic trait in yeast. Science 273: 622.

Paushkin S.V., Kushnirov V.V., Smirnov V.N., and TerAvanesyan M.D. 1996. Propagation of the yeast prion-like $\left[\mathrm{psi}^{+}\right]$determinant is mediated by oligomerization of the $S U P 35$-encoded polypeptide chain release factor. EMBO J. 15: 3127.

Petkova A.T., Ishii Y., Balbach J.J., Antzutkin O.N., Leapman R.D., Delaglio F., and Tycko R. 2002. A structural model for Alzheimer's beta-amyloid fibrils based on experimental constraints from solid state NMR. Proc. Natl. Acad. Sci. 99: 16742.

Priola S.A., Chesebro B., and Caughey B. 2003. Biomedicine. A view from the top-Prion diseases from 10,000 feet. Science 300: 917.

Prusiner S.B. 1982. Novel proteinaceous infectious particles cause scrapie. Science 216: 136.

Rai R., Tate J.J., and Cooper T.G. 2003. Ure2, a prion precursor with homology to glutathione S-transferase, protects Saccharomyces cerevisiae cells from heavy metal ion and oxidant toxicity. J. Biol. Chem. 278: 12826.

Resende C., Parham S.N., Tinsley C., Ferreira P.C., Duarte
J.A.B., and Tuite M.F. 2002. The Candida albicans Sup35p protein (CaSup35p): Function, prion-like behavior and an associated polyglutamine length polymorphism. Microbiology 148: 1049.

Riggs A.D. and Porter T.N. 1997. Overview of epigenetic mechanisms. In Epigenetic mechanisms of gene regulation (ed. V.E.A. Russo et al.), p. 29. Cold Spring Harbor Laboratory Press, Cold Spring Harbor, New York.

Roberts B.T., Moriyama H., and Wickner R.B. 2003. [URE3] prion propagation is abolished by a mutation of the primary cytosolic Hsp70 of budding yeast. Yeast 21: 107.

Roberts B.T. and Wickner R.B. 2004. A class of prions that propagate via covalent auto-activation. Genes Dev. 17: 2083.

Ross E.D., Baxa U., and Wickner R.B. 2004. Scrambled prion domains form prions and amyloid. Mol. Cell. Biol. 24: 7206.

Saupe S.J., Clave C., and Begueret J. 2000. Vegetative incompatibility in filamentous fungi: Podospora and Neurospora provide some clues. Curr. Opin. Microbiol. 3: 608.

Schwimmer C. and Masison D.C. 2002. Antagonistic interactions between yeast [PSI+] and [URE3] prions and curing of [URE3] by Hsp70 protein chaperone Ssa1p but not by Ssa2p. Mol. Cell. Biol. 22: 3590.

Silar P. and Daboussi M.J. 1999. Non-conventional infectious elements in filamentous fungi. Trends Genet. 15: 141.

Silar P., Haedens V., Rossingnol M., and Lalucque H. 1999. Propagation of a novel cytoplasmic, infectious and deleterious determinant is controlled by translational accuracy in $\mathrm{Po}$ dospora anserina. Genetics 151: 87.

Singh A.C., Helms C., and Sherman F. 1979. Mutation of the non-Mendelian suppressor $\psi^{+}$in yeast by hypertonic media. Proc. Natl. Acad. Sci. 76: 1952.

Sondheimer N. and Lindquist S. 2000. Rnq1: An epigenetic modifier of protein function in yeast. Mol. Cell 5: 163.

Speransky V., Taylor K.L., Edskes H.K., Wickner R.B., and Steven A. 2001. Prion filament networks in [URE3] cells of Saccharomyces cerevisiae. J. Cell Biol. 153: 1327.

Stansfield I., Jones K.M., Kushnirov V.V., Dagkesamanskaya A.R., Poznyakovski A.I., Paushkin S.V., Nierras C.R., Cox B.S., Ter-Avanesyan M.D., and Tuite M.F. 1995. The products of the SUP45 (eRF1) and SUP35 genes interact to mediate translation termination in Saccharomyces cerevisiae. EMBO J. 14: 4365.

Taylor K.L., Cheng N., Williams R.W., Steven A.C., and Wickner R.B. 1999. Prion domain initiation of amyloid formation in vitro from native Ure2p. Science 283: 1339.

Teichert U., Mechler B., Muller H., and Wolf D.H. 1989. Lysosomal (vacuolar) proteinases of yeast are essential catalysts for protein degradation, differentiation, and cell survival. $J$. Biol. Chem. 264: 16037.

Ter-Avanesyan A., Dagkesamanskaya A.R., Kushnirov V.V., and Smirnov V.N. 1994. The SUP35 omnipotent suppressor gene is involved in the maintenance of the non-Mendelian determinant [psi+] in the yeast Saccharomyces cerevisiae. Genetics 137: 671 .

Tuite M.F. and Cox B.S. 2003. Propagation of yeast prions. Nat. Rev. Mol. Cell Biol. 4: 878 .

Tycko R. 2003. Insights into the amyloid folding problem from solid-state NMR. Biochemistry 42: 3151.

Umland T.C., Taylor K.L., Rhee S., Wickner R.B., and Davies D.R. 2001. The crystal structure of the nitrogen catabolite regulatory fragment of the yeast prion protein Ure2p. Proc. Natl. Acad. Sci. 98: 1459.

Wickner R.B. 1994. Evidence for a prion analog in S. cerevisiae: The [URE3] non-Mendelian genetic element as an altered URE2 protein. Science 264: 566.

Wickner R.B., Edskes H.K., Ross E.D., Pierce M.M., Baxa U., Brachmann A., and Shewmaker F. 2004. Prion genetics: New rules for a new kind of gene. Annu. Rev. Genet. 38: 681.

Zubenko G.S., Park F.J., and Jones E.W. 1982. Genetic properties of mutations at the PEP4 locus in Saccharomyces cerevisiae. Genetics 102: 679. 


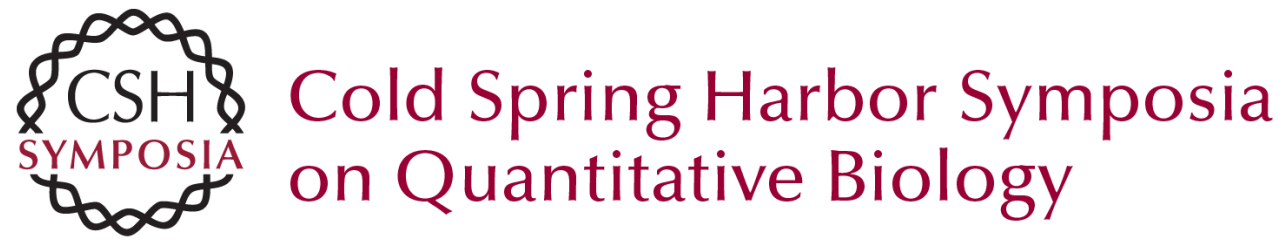

\section{Prions of Yeast Are Genes Made of Protein: Amyloids and Enzymes}

R.B. WICKNER, H.K. EDSKES, E.D. ROSS, et al.

Cold Spring Harb Symp Quant Biol 2004 69: 489-496

Access the most recent version at doi:10.1101/sqb.2004.69.489

References This article cites 77 articles, 46 of which can be accessed free at: http://symposium.cshlp.org/content/69/489.full.html\#ref-list-1

License

Email Alerting Receive free email alerts when new articles cite this article - sign up in Service the box at the top right corner of the article or click here. 